\title{
Constructing a Continuous Phase Time History from TDMA Signals for Opportunistic Navigation
}

\author{
Kenneth M. Pesyna, Jr. and Zaher M. Kassas \\ Department of Electrical and \\ Computer Engineering \\ The University of Texas at Austin \\ Email: kpesyna@utexas.edu, zkassas@ieee.org
}

\author{
Todd E. Humphreys \\ Department of Aerospace Engineering and \\ Engineering Mechanics \\ The University of Texas at Austin \\ Email: todd.humphreys@mail.utexas.edu
}

\begin{abstract}
A technique is developed for reconstructing a continuous phase time history from the noncontinuous phase bursts of time division multiple access (TDMA) signals. A continuous phase time history facilitates exploitation of TDMA signals as signals of opportunity (SOPs) within an opportunistic navigation framework. Because of their widespread use and availability in today's wireless communication market, TDMA signals are attractive candidate SOPs for opportunistic navigation. The phase reconstruction technique presented here combines an integer least squares technique for estimating phase ambiguities at the beginning of each TDMA phase burst with a Kalman filter and smoother for removing these ambiguities and optimally "stitching" the bursts together. A Monte-Carlo-type simulation and test environment has been developed to investigate the sensitivity of the proposed phase reconstruction technique to various system parameters, namely, carrier-to-noise ratio, receiver clock quality, TDMA transmitter clock quality, line-of-sight acceleration uncertainty, and TDMA burst structure. Simulation results indicate that successful carrier phase reconstruction is most strongly dependent on the TDMA burst period and on the combined phase random walk effect of the receiver and transmitter clocks, the propagation effects, and the range errors.
\end{abstract}

\section{INTRODUCTION}

The pervasion of ambient radio-frequency signals in today's urban and indoor environments has spurred research in the area of hybrid signal navigation [1]-[9]. One hybrid navigation technique, known as opportunistic navigation [2], calls for a centralized estimator to ingest pseudorange and carrier phase observables from heterogeneous wireless signals to compute a receiver's position, velocity, and time. TDMA signals are a prime candidate for opportunistic navigation because they are widely available due to their adoption into many terrestrial and satellite wireless communication standards. However, the intermittent signal availability and phase ambiguities resulting from the time-multiplexed signal structure makes it challenging to incorporate raw TDMA observables into carrier-phasebased navigation and timing algorithms.

Phase ambiguities arise in TDMA signal tracking because the receiver cannot track the carrier phase evolution between TDMA transmission slots (hereafter bursts) and because the receiver's phase discriminator is only capable of measuring phase to within 1 cycle. As a result, there is an integer cycle ambiguity at the beginning of each burst which must be resolved before navigation and timing information can be fully extracted from the TDMA signals. Some TDMA systems introduce a further complication by randomizing the initial phase of a burst so that for practical purposes it becomes fractional-cycle ambiguous (i.e., the ambiguity is imposed in increments of $\frac{1}{M}$ cycles, where $M \geq 2$ is an integer). Fractional-cycle ambiguities are naturally more difficult to resolve than whole-cycle ambiguities $(M=1)$.

To fully exploit TDMA signals in an opportunistic navigation framework, a technique is needed for reconstructing a continuous carrier phase time history from a TDMA signal's noncontinuous bursts. No such technique has been offered previously in the literature, as far as the authors are aware. This paper's contributions are twofold: (1) An optimal technique for TDMA phase reconstruction is developed, and (2) the performance of the proposed technique and the technique's sensitivity to parameters of practical interest is analyzed.

This paper is organized as follows. Section II sets up the system model, motivating the need for and the effects of carrier phase reconstruction. Section III presents the reconstruction technique. The technique addresses the two main challenges in exploiting TDMA signals for navigation: (1) their burst structure and (2) the whole- or fractional-cycle phase ambiguities present at the beginning of each burst. Section IV presents a simulation and test environment designed to simulate TDMA signals, apply the reconstruction technique, and then evaluate its performance. Section V discusses the technique's performance by analyzing its sensitivity to different simulation parameters. Concluding remarks are given in Section VI.

\section{CARrier Phase Models}

Two carrier phase models are introduced here to illustrate the need for and the effects of carrier phase reconstruction: (1) the residual TDMA carrier phase model, which models the signal before the phase reconstruction technique is applied, and (2) the smoothed reconstructed carrier phase model, which models the signal after the reconstruction technique is applied.

\section{A. Residual TDMA Carrier Phase Model}

Let the residual carrier phase $\phi_{r}(t)$ be defined as the measured difference between the received carrier phase and the phase of the local signal replica, which is the receiver's best prediction of received carrier phase. The term "residual" 
refers to this being the phase remaining after downmixing and correlation with the local signal replica. The residual carrier phase in cycles can be modeled by the following adaptation of the carrier phase measurement model given in [10]:

$\phi_{r}(t)=$ received carrier phase - predicted carrier phase

$$
=\left\{\begin{array}{cc}
\frac{1}{\lambda} r_{e}(t)+\frac{c}{\lambda}\left[\delta t_{R X}(t)-\delta t_{T X}(t)\right]+\gamma_{0}-\psi_{0} \\
+\epsilon_{p}(t)+v_{\phi}(t)+\frac{1}{M} \eta(t) & \text { for } t_{s i} \leq t \leq t_{s i}+T_{b} \\
& i=0,1, \ldots, N_{b}-1 \\
\text { undefined } & \text { otherwise }
\end{array}\right.
$$

with the following definitions:

$\lambda$ the TDMA carrier wavelength, in meters.

$r_{e} \quad$ the error in the predicted range between the receiver and transmitter, in meters.

$c \quad$ the speed of light, in meters per second.

$\delta t_{R X}$ the receiver clock error, in seconds.

$\delta t_{T X}$ the TDMA transmitter clock error, in seconds.

$\gamma_{0}$ the phase of the receiver's carrier replica at the time of first acquisition, in cycles.

$\psi_{0} \quad$ the phase of the transmitted TDMA signal at the time of first acquisition, in cycles.

$\epsilon_{p} \quad$ the carrier phase deviation due to atmospheric and multipath effects, in cycles.

$v_{\phi} \quad$ the measurement noise induced by the receiver frontend, in cycles.

$\frac{1}{M} \quad$ the ambiguity factor used to depict whole-cycle phase ambiguities $(M=1)$ or fractional-cycle phase ambiguities $(M>1)$, whichever is appropriate for the signal being modeled.

$\eta(t) \quad$ an integer constant, measured in cycles. Upon scaling by $\frac{1}{M}$, it represents the offset of the signal's measured phase from that of the unambiguous "ideal" phase at the beginning of each burst. In particular, $\eta(t)=n_{i}$ is constant when $t$ is within the time spanned by the $i$ th burst. In this paper, $\eta(t)$ will often be referred to as the "integer ambiguity."

$t_{s i}$ the start time of the $i^{t h}$ burst, in seconds.

$T_{b}$ the TDMA burst duration, in seconds.

$N_{b} \quad$ the number of bursts.

The TDMA reconstruction technique reconstructs a continuous phase time history from the burst-like structure of $\phi_{r}(t)$. Figs. 1-4 help to build intuition about $\phi_{r}(t)$ and about the challenges of phase reconstruction. Fig. 1 illustrates how $\phi_{r}(t)$ can be measured only during the periodic TDMA bursts even though the underlying transmitter and receiver clocks maintain phase continuity between bursts. $T_{p}$ represents the burst period, i.e., the time between consecutive bursts, and $T_{b}$ represents the burst duration. The dotted lines represent the true but unmeasurable value of $\phi_{r}(t)$ between bursts. The illustration represents a fictitious scenario in which the measurements of $\phi_{r}(t)$, represented by the solid black curves within each burst, suffer from no phase ambiguity. In reality, due to (1) the unavailability of carrier phase measurements between bursts, (2) the insensitivity of the phase discriminator to integer cycle offsets in phase, and, in some cases, (3)

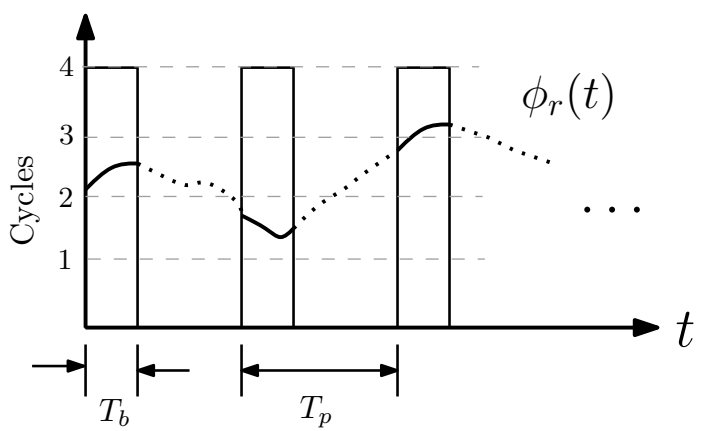

Fig. 1. Illustration of residual carrier phase measurements $\phi_{r}(t)$ made by a receiver during each burst. Also shown is the underlying continuous phase time history, which must be reconstructed between each burst. This scenario is fictitious, however, because the phase measurements suffer from no phase ambiguities as introduced in Figs. 2 and 3.

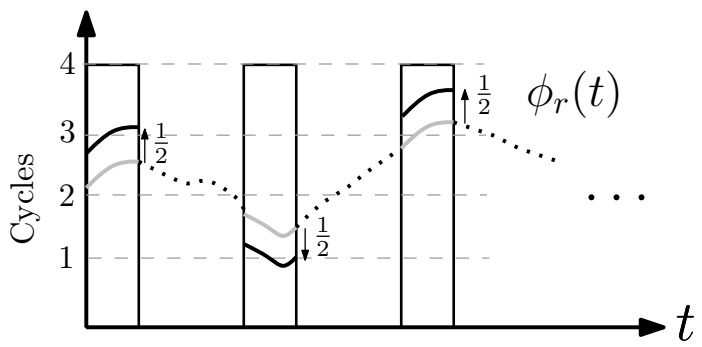

Fig. 2. Illustration of the residual carrier phase measurements $\phi_{r}(t)$ made by the receiver during each burst. In this situation, random phase offsets to the underlying phase are imposed by the transmitter. These offsets will need to be resolved when reconstructing the continuous phase time history.

a random $\frac{1}{M}$-cycle phase shift imposed by the transmitting system on the phase at the beginning of each burst, the actual measurements of $\phi_{r}(t)$ are quite different from what is suggested by the solid curves in Fig. 1.

Fig. 2 introduces the random $\frac{1}{M}$-cycle phase shifts that are imposed by some transmitting TDMA systems on the phase at the beginning of each burst. In order to accurately reconstruct the underlying continuous phase time history, these shifts will need to be determined and accounted for during reconstruction. In the case shown, $M=2$, resulting in random $\frac{1}{2}$-cycle shifts. Within the residual carrier phase model (1), these transmitterimposed offsets have been modeled by the $\frac{1}{M} \eta(t)$ term. This term also represents the phase aliasing effect caused by the insensitivity in the receiver's phase discriminator to wholecycle phase shifts.

Fig. 3 introduces the effect of phase aliasing caused by the insensitivity of the receiver's phase discriminator to whole-cycle phase shifts. Illustrated is the combined effect of this whole-cycle phase aliasing with the fractional-cycle transmitter-imparted phase shifts. The phase at the start of each burst is aliased into the region $\left[0, \frac{1}{M}\right)$, where $M=2$ in the case shown. This combined effect is referred to as a "fractional-cycle phase ambiguity" or simply "phase ambiguity" hereafter. Fig. 3 accounts for all subtleties arising from TDMA signals and is a realistic depiction of the receiver's residual phase measurements $\phi_{r}(t)$. 


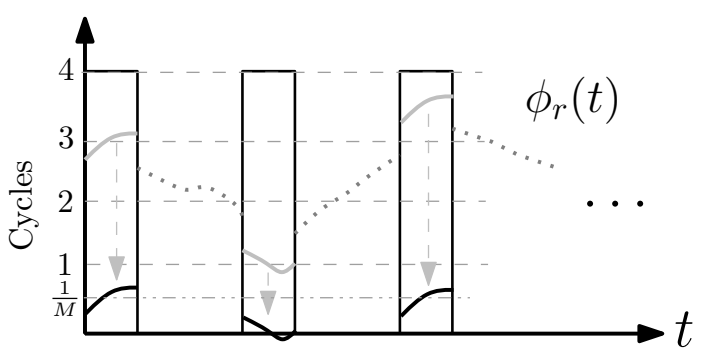

Fig. 3. Illustration of the residual carrier phase measurements $\phi_{r}(t)$ made by the receiver during each burst. The solid gray curves represent the fractionalcycle phase shifts imposed the transmitter which then become aliased between 0 and $\frac{1}{M}$ cycles due to a combination of these shifts and the insensitivity of the receiver's phase detector to whole-cycle phase offsets. The final received phase with transmitter imposed offsets and subsequent aliasing is represented by the solid black curves and is a realistic depiction of the receiver's measurements of $\phi_{r}(t)$.

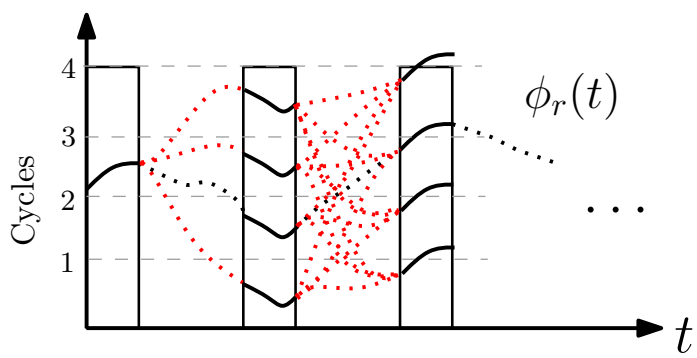

Fig. 4. Possible phase trajectories, only 1 of which corresponds to the underlying truth. It is the job of the reconstruction algorithm to select the correct trajectory from its measurements of $\phi_{r}(t)$, which are ambiguous in this scenario to 1 cycle.

Fig. 4 depicts the effect of phase ambiguities on phase reconstruction. In this illustration, $M=1$, corresponding to whole-cycle ambiguities. Because the receiver only has access to the ambiguous $\phi_{r}(t)$ as represented by the solid black lines in Fig. 3, the reconstruction algorithm must determine in which whole-cycle region (or fractional-cycle region if instead $M>1$ ) each solid black curve would reside if $\phi_{r}(t)$ was unambiguous (as presented in Fig. 1). The solid curved lines in each burst of Fig. 4 represent possible true values of the phase in each burst. These ambiguities extend infinitely in each direction. This leads to an infinite number of phase trajectories, the 16 most probable of which are depicted here. Only one of the possible trajectories accurately depicts the so-called ideal phase time history $\phi_{\text {ideal }}(t)$. The ideal phase time history represents the residual carrier phase $\phi_{r}(t)$ without the phase dropouts due to the burst structure, without the phase aliasing effect, without the transmitterimposed phase shifts, and without measurement noise. It is the task of the reconstruction algorithm to use past, present, and future measurements of $\phi_{r}(t)$ to resolve the phase ambiguities and attempt to accurately reconstruct $\phi_{\text {ideal }}(t)$. If a phase ambiguity is resolved incorrectly, this would lead to an incorrect reconstructed phase trajectory, limiting the signal's usefulness in an opportunistic navigation framework [2], [11].

\section{B. Smoothed Reconstructed TDMA Carrier Phase Model}

Let the smoothed reconstructed carrier phase $\phi_{s}(t)$ be defined as the carrier phase after applying forward-pass Kalman filtering, optimal integer ambiguity resolution, and backwardpass smoothing on $\phi_{r}(t)$. The smoothed reconstructed carrier phase is the receiver's best estimate of $\phi_{\text {ideal }}(t)$, the noise-free and ambiguity-free beat carrier phase. $\phi_{s}(t)$ can be modeled as follows:

$$
\begin{aligned}
\phi_{s}(t)= & \frac{1}{\lambda} r_{e}(t)+\frac{c}{\lambda}\left[\delta t_{R X}(t)-\delta t_{T X}(t)\right]+\gamma_{0}-\psi_{0} \\
& +\epsilon_{p}(t)+v_{\phi_{s}}(t)+\frac{1}{M}\left[\eta(t)-\hat{\eta}_{s}(t)\right]
\end{aligned}
$$

with the following new definitions:

$\hat{\eta}_{s}(t)$ the receiver's best estimate of the time-varying integer ambiguity term $\eta(t)$ after forward-pass filtering and subsequent ambiguity resolution.

$v_{\phi_{s}} \quad$ the smoothed measurement noise after forward-pass filtering, ambiguity resolution, and backward-pass smoothing.

\section{Reconstruction Technique}

This section presents a reconstruction technique designed to address the two main challenges in exploiting TDMA signals for navigation: (1) their burst structure and (2) the whole- or fractional-cycle phase ambiguities present at the beginning of each burst. These two challenges are addressed through the use of an integer least-squares solver integrated with a Kalman filter and smoother. A square-root information implementation of the filter and smoother are used to perform the phase reconstruction of TDMA signals in an accurate and computationally efficient manner [12], [13]. This section discusses the structure of the filter and smoother as well as the method used to estimate the integer phase ambiguities.

\section{A. State Dynamics and Measurement Model}

The dynamics of the noise-free residual carrier phase can be modeled in discrete-time as a state-space system with a mixed real and integer state. The real part of the state evolves as a first-order Gauss-Markov process with process noise representing the variations due to $r_{e}(t), \delta t_{R X}(t), \delta t_{T X}(t)$, and $\epsilon_{p}(t)$ in (1). The integer part of the state evolves under the assumption that a new constant integer ambiguity is introduced with each burst. The measurement of $\phi_{r}(t)$ is modeled in discrete time in a way that relates the integer ambiguities in the state to the phase ambiguities inherent in the phase measurements obtained during each burst.

1) State: The real-valued state at time $t_{k}$ can be expressed as

$$
\mathbf{x}_{k}=\left[\phi_{k}, \omega_{k}\right]^{\top}
$$

with the following definitions:

$\phi_{k} \quad$ the noise- and ambiguity-free residual carrier phase at time $t_{k}$, in cycles.

$\omega_{k}$ the rate of change of the noise- and ambiguity-free residual carrier phase at time $t_{k}$, in $\mathrm{Hz}$. 
The integer-valued state $\mathbf{n}_{k}$ at time $t_{k}$ can be expressed as

$$
\mathbf{n}_{k}=\left[n_{1}, n_{2}, \ldots, n_{i_{k}}\right]^{\top}
$$

with the following definitions:

$\mathbf{n}_{k} \quad$ an $i_{k}-$ by- 1 vector of integers, one for each burst that began between time 0 and time $t_{k}$.

$n_{i_{k}} \quad$ the integer corresponding to the $i_{k}^{\text {th }}$ burst, the mostrecent burst beginning at or before time $t_{k}$.

2) Dynamics Model: The following linear model describes the time evolution of the mixed real/integer state:

$$
\begin{aligned}
& \mathbf{x}_{k+1}=\Phi \mathbf{x}_{k}+\Gamma \mathbf{w}_{k}, \quad \mathbf{w}_{k} \sim \mathcal{N}(\mathbf{0}, Q) \\
& \mathbf{n}_{k+1}= \begin{cases}{\left[\begin{array}{c}
\mathbf{n}_{k} \\
n_{i_{k+1}}
\end{array}\right]} & \begin{array}{c}
\text { if a new burst began within the } \\
\text { interval }\left(t_{k}, t_{k+1}\right]
\end{array} \\
{\left[\mathbf{n}_{k}\right]} & \text { otherwise }\end{cases}
\end{aligned}
$$

with the following definitions:

$$
\begin{array}{ll}
\Phi & \text { the real-valued state transition matrix. } \\
\Gamma & \text { the process noise influence matrix. } \\
\mathbf{w}_{k} & \text { the process noise at time } t_{k} . \\
Q & \text { the process noise covariance matrix. }
\end{array}
$$

The state transition matrix for the real-valued state models standard Euler integration from $t_{k}$ to $t_{k+1}$ :

$$
\Phi=\left[\begin{array}{ll}
1 & T \\
0 & 1
\end{array}\right]
$$

Here, $T=t_{k+1}-t_{k}$ represents the time interval between consecutive filter updates. The process noise influence matrix is given by

$$
\Gamma=\left[\begin{array}{ll}
1 & 0 \\
0 & 1
\end{array}\right]
$$

and the process noise covariance matrix by

$$
Q=S_{g} \omega_{0}^{2}\left[\begin{array}{cc}
\frac{T^{3}}{3} & \frac{T^{2}}{2} \\
\frac{T^{2}}{2} & T
\end{array}\right]+S_{f} \omega_{0}^{2}\left[\begin{array}{cc}
T & 0 \\
0 & 0
\end{array}\right]
$$

where $\omega_{0}$ is the TDMA signal's nominal carrier frequency, in cycles per second. The parameters $S_{f}$ and $S_{g}$ model the combined phase instability in the transmitter and receiver clocks and the "clock-like" phase instability caused by $r_{e}(t)$ and $\epsilon_{p}(t)$. They are defined as [14]

$$
\begin{aligned}
S_{f} & =\frac{h_{0}}{2} \\
S_{g} & =2 \pi^{2} h_{-2}
\end{aligned}
$$

where $h_{0}$ and $h_{-2}$ are parameters characterizing the power spectral density of the combined phase instability. These " $h$ parameters" are further defined and discussed in Sec. IV-A.
3) Measurement Model: The residual phase model from (1) is represented in discrete time and as a function of the real and integer state components $\mathbf{x}_{k}$ and $\mathbf{n}_{k}$ by

$\phi_{r k}=\left\{\begin{array}{lc}\tilde{H}_{x k} \mathbf{x}_{k}+\tilde{H}_{n k} \mathbf{n}_{k}+v_{\phi k} & \text { for } t_{s i} \leq t_{k} \leq t_{s i}+T_{b} \\ & i=0,1, \ldots, N_{b}-1 \\ \text { undefined } & \text { otherwise }\end{array}\right.$

with the following definitions:

$\phi_{r k}$ the phase measurement in cycles at time $t_{k}$ made within the $i_{k}^{t h}$ burst.

$\tilde{H}_{x k}$ the measurement sensitivity matrix for the realvalued state at time $t_{k}$.

$\tilde{H}_{n k}$ the measurement sensitivity matrix for the integervalued state at time $t_{k}$.

$v_{\phi k}$ the measurement noise at time $t_{k}$, modeled as a zeromean discrete-time Gaussian white noise process, $v_{\phi k} \sim \mathcal{N}\left(0, \sigma_{\phi k}^{2}\right)$

$\sigma_{\phi k}^{2} \quad$ the measurement noise variance at time $t_{k}$.

The quantities $\mathbf{x}_{k}$ and $\mathbf{n}_{k}$ are as previously described. The measurement sensitivity matrices can be expanded as

$$
\begin{aligned}
\tilde{H}_{x k} & =\left[\begin{array}{ll}
1 & 0
\end{array}\right] \\
\tilde{H}_{n k} & =\left[0,0, \ldots, 0, \frac{1}{M}\right]
\end{aligned}
$$

where $\frac{1}{M}$ is the ambiguity factor defined previously. Two features of the $1-$ by $-i_{k}$ matrix $\tilde{H}_{n k}$ are noteworthy. First, the $\frac{1}{M}$ factor in its last element allows the integer-valued state $\mathbf{n}_{k}$ to model whole-cycle phase ambiguities $(M=1)$ or fractional-cycle phase ambiguities $(M>1)$. Second, $\tilde{H}_{n k}$ has $0 \mathrm{~s}$ in all but its last element to ensure that the measurement at time $t_{k}$ is only affected by the most recent integer ambiguity $n_{i_{k}}$ in $\mathbf{n}_{k}$.

\section{B. Cost Function}

Optimal estimates of the state components $\mathbf{x}_{k}$ and $\mathbf{n}_{k}$ according to the maximum a posteriori criterion and based on all measurements $\phi_{r k}$ from $k=0$ to $K$ can be found by determining the state and process noise time histories that minimize a certain cost function subject to the dynamics model. For numerical robustness, a square-root-information approach is adopted [12], [13]. Let the square-root information equation for the a priori real-valued state estimate at $k=0$ be given by

$$
\mathbf{z}_{x 0}=R_{x x 0} \mathbf{x}_{0}+\mathbf{w}_{x 0}, \quad \mathbf{w}_{x 0} \sim \mathcal{N}(\mathbf{0}, \mathrm{I}) .
$$

No a priori information is assumed to be available at $\mathrm{k}=0$ for the integer-valued state component $\mathbf{n}_{0}$. Let the square-root information equation for the $a$ priori process noise estimate at $\mathrm{k}$ be

$$
\mathbf{z}_{w 0}=0=R_{w w 0} \mathbf{w}_{k}+\mathbf{w}_{w k}, \quad \mathbf{w}_{w k} \sim \mathcal{N}(\mathbf{0}, \mathrm{I}) .
$$

Also, let the measurement model in (12) be transformed by multiplying both sides by $\sigma_{\phi k}^{-1}$. The transformed measurement 
model is written as

$$
z_{k}=H_{x k} \mathbf{x}_{k}+H_{n k} \mathbf{n}_{k}+v_{z k}, \quad v_{z k} \sim \mathcal{N}(0,1) .
$$

Like $\phi_{r k}$ in (12), $z_{k}$ is undefined between bursts.

Given these transformations, the optimal phase reconstruction problem can be expressed as follows:

Find $\mathbf{x}_{k}$ for $k=0,1, \ldots, K, \mathbf{w}_{k}$ for $k=0,1, \ldots, K-1$, and $\mathbf{n}_{K}=\left[n_{0}, n_{1}, \ldots, n_{i_{K}}\right]$ to minimize

$$
\begin{aligned}
J= & \left\|R_{x x 0} \mathbf{x}_{0}-z_{x 0}\right\|^{2}+\sum_{k=0}^{K-1}\left\|R_{w w k} \mathbf{w}_{k}\right\|^{2} \\
& +\sum_{k=0}^{K}\left\|H_{x k} \mathbf{x}_{k}+H_{n k} \mathbf{n}_{K}-z_{k}\right\|^{2}
\end{aligned}
$$

subject to the dynamics equations (5) and (6).

A solution can be readily found by breaking the problem into three stages: forward-pass filtering, ambiguity resolution, and backward-pass smoothing. It can be shown that in solving the forward-pass filtering problem, the mixed integer/real cost function presented in (18) can be cast as a series of mixed integer/real linear least squares problems, one at each index $k$. In particular, the problem can be cast as a sum of three independent terms [15]: (1) a term involving both the realand integer-valued states $\mathbf{n}_{k}$ and $\mathbf{x}_{k},(2)$ a term only having to do with the integer part of the state, $\mathbf{n}_{k}$, and (3) an irreducible residual:

$$
\begin{aligned}
J_{\text {mixed }}\left(\mathbf{x}_{k}, \mathbf{n}_{k}\right)= & \underbrace{\left\|R_{x x k} \mathbf{x}_{k}+R_{x n k} \mathbf{n}_{k}-\mathbf{z}_{x k}\right\|^{2}}_{\text {Term involving the integer- and real-valued states }} \\
+ & \underbrace{\left\|R_{n n k} \mathbf{n}_{k}-\mathbf{z}_{n k}\right\|^{2}}_{\text {Term involving only the integer-valued state }}+\underbrace{\left\|\mathbf{z}_{r k}\right\|^{2}}_{\text {Residual term }}
\end{aligned}
$$

with the following definitions:

$\mathbf{z}_{x k}$ the real-state nonhomogeneous term at time $t_{k}$.

$\mathbf{z}_{n k}$ the integer-state nonhomogeneous term at time $t_{k}$.

$\mathbf{z}_{r k}$ the residual nonhomogeneous term at time $t_{k}$.

$R_{x x k}$ the square-root information matrix at time $t_{k}$.

$R_{n n k}$ the square-root ambiguity information matrix at time $t_{k}$.

$R_{x n k}$ the square-root ambiguity/state information matrix at time $t_{k}$.

The above terms are in square-root information form and are outputs of the square root information filter (SRIF) at each time step. For details on these terms and their structure, see [13].

To minimize (19), it is appropriate to first determine the integer-valued vector state estimate $\hat{\mathbf{n}}_{k}$ that minimizes the second term, that is, the term involving only the integer-valued state. This estimate can be determined efficiently using integer least-squares techniques. It can then be inserted into the first term, that is, the one involving both the integer- and realvalued states. At this point, it is possible to determine the real-valued state estimate $\hat{\mathbf{x}}_{k}$ that minimizes this first term.

\section{Forward-Pass Filtering}

Intermediate equations of the form in (19) are produced by processing the measurements $z_{k}$ for $k=0,1, \ldots, K$ through a forward-pass SRIF. The SRIF also stores up information regarding the value of the integer ambiguities.

\section{Ambiguity Resolution}

The real- and integer-valued state vectors $\mathbf{x}_{k}$ and $\mathbf{n}_{k}$ that minimize (19) can be found by first finding the vector of integer ambiguities $\mathbf{n}_{k}$ that minimizes the second term of (19):

$$
J_{n}\left(\mathbf{n}_{k}\right)=\left\|R_{n n k} \mathbf{n}_{k}-\mathbf{z}_{n k}\right\|^{2}
$$

To resolve the integer ambiguities, the least-squares ambiguity decorrelation adjustment method (LAMBDA) is used [16], [17]. This algorithm accepts the integer-state information matrix $R_{n n K}$ and the integer-state nonhomogeneous term $\mathbf{z}_{n K}$ from the forward-pass SRIF after the last measurement $K$ has been ingested at time $t_{K}$ and selects the vector of integer ambiguities $\hat{\mathbf{n}}_{K}$ that minimizes (20). It is important to note here that there exist many techniques for minimizing (20), including a brute-force search among all remotely possible $\mathbf{n}_{k}$. The LAMBDA method is just an efficient way of doing this.

\section{E. Backward-Pass Smoothing}

Backward-pass smoothing is the third step in the phase reconstruction process. The smoothed state estimates $\mathbf{x}_{k}^{\star}$ for $k=0,1, \ldots, K$ can be expressed in square-root information form as

$$
\mathbf{x}_{k}^{\star}=\left(R_{x x k}^{\star}\right)^{-1} \mathbf{z}_{x k}^{\star}
$$

with the following definitions:

$R_{x x k}^{\star}$ the smoothed square-root information matrix at time $t_{k}$.

$z_{x k}^{\star} \quad$ the smoothed nonhomogeneous term at time $t_{k}$.

To initialize the smoother, the integer ambiguity vector estimate $\hat{\mathbf{n}}_{K}$ at time $t_{K}$ is determined as described above and then incorporated into the smoother's initial nonhomogeneous term $\mathbf{z}_{x K}^{\star}$ and initial square-root information matrix $R_{x n K}^{\star}$ by

$$
\begin{aligned}
\mathbf{z}_{x K}^{\star} & =\mathbf{z}_{x K}-R_{x n K} \mathbf{n}_{K} \\
R_{x n K}^{\star} & =R_{x n K}
\end{aligned}
$$

where

$K \quad$ is the last time index processed by the forward-pass filter,

$\mathbf{z}_{x K}$ is the filter's nonhomogeneous term at time $t_{K}$, and $R_{x n K}$ is the filter's square-root information matrix at time $t_{K}$.

At time $t_{K}$ the smoother begins its backwards-pass processing, working backward to smooth the filtered state estimate at each time step until it reaches $k=0$. 


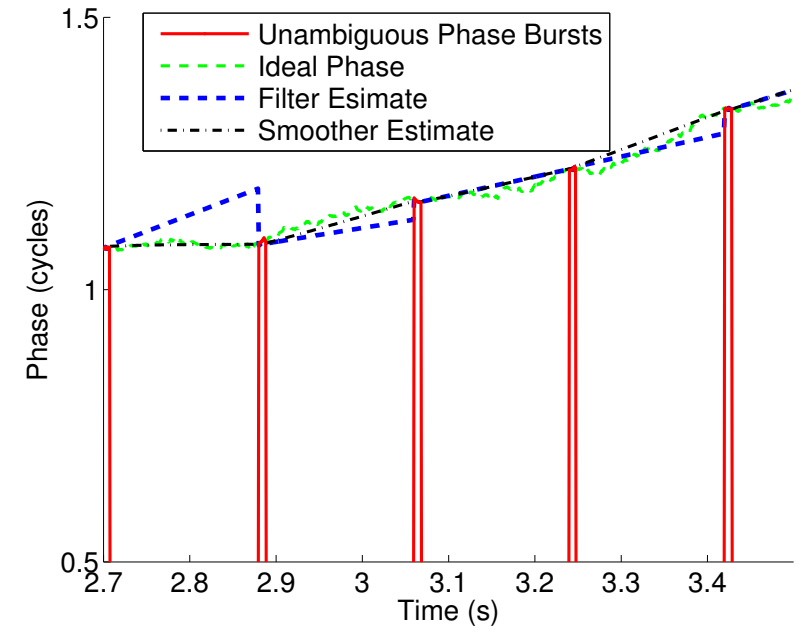

Fig. 5. Illustration of the abrupt dynamics possible in the filter's phase estimates as compared to the smoothed dynamics in the smoother's phase estimates.

\section{F. Discussion on Smoothing}

Given that backward-pass smoothing is noncausal and so prevents real-time implementation, one may wonder whether smoothing is necessary for phase reconstruction. There are, in fact, scenarios for which smoothing is useful. For example, if the reconstruction technique is used in an opportunistic navigation application that seeks to estimate the underlying noise-free and ambiguity-free continuous carrier phase $\phi_{k}$ with sufficient fidelity to enable extended coherent integration of weak SOPs, it may be necessary to use smoothed as opposed to filtered estimates of $\phi_{k}$. This is because the innovations introduced during forward-pass filtering cause discontinuities in $\left\{\hat{\phi}_{k}, k=0,1, \ldots, K\right\}$ that degrade its coherence, which can be expressed by the discrete-time coherence function

$$
C_{\text {coh }}(K)=\left|\frac{1}{K} \sum_{k=0}^{K-1} e^{j \tilde{\phi}_{k}}\right| \quad 0 \leq C_{\text {coh }}(K) \leq 1
$$

where $\tilde{\phi}_{k}=\phi_{k}-\hat{\phi}_{k}$. The so-called coherence time, which is the duration over which phasors of the form $e^{j \phi_{k}}$ can be coherently summed, can be defined as $\tau_{\text {coh }}=T \cdot K_{\text {coh }}$, where $T$ is the update interval and $K_{\text {coh }}$ is the value of $K$ at which $\mathbb{E}\left[C_{\text {coh }}^{2}(K)\right]$ drops below 0.5 [18]. If instead the smoothed estimates of $\phi_{k}$ are used so that $\tilde{\phi}_{k}=\phi_{k}-\phi_{k}^{*}$, then the coherence time $\tau_{\text {coh }}$ is increased because the effect of backward smoothing is to force the sequence of estimates $\left\{\phi_{k}^{\star}, k=0,1, \ldots, K\right\}$ to conform to the dynamics model (5), thereby smoothing the discontinuities introduced by measurement innovations in forward-pass filtering.

Fig. 5 illustrates the benefits of smoothing. As shown by the thick dashed trace, new measurements at the beginning of each burst introduce innovations within the SRIF that create sharp phase corrections when the phase estimates drift significantly between bursts. These corrections lead to abrupt phase dynamics that may not conform to the filter's state dynamics model, even when the filter's assumed dynamics and measurement models are an accurate reflection of reality. As shown by the thinner dash-dotted trace, the smoother, because it has knowledge of future measurements, is able to remove these unrealistic dynamics introduced by innovations in the SRIF. In other words, smoothing more accurately recreates the actual signal dynamics, causing the state variations to conform more closely to the a priori dynamics model, and, consequently, to the true state, thereby increasing coherence time.

\section{Simulation and Test EnVironment}

To test the performance of the reconstruction technique, a Monte-Carlo-type simulation and test environment has been designed in MATLAB. The environment performs two tasks. First, using models of the error sources, it simulates the residual carrier phase $\phi_{r}(t)$ of TDMA signals, a model for which was introduced in Sec. II-A. Parameters accurately modeling the the line-of-sight acceleration uncertainty, receiver clock quality, transmitter clock quality, propagationinduced effects, and carrier-to-noise ratio can be input into the simulator. TDMA burst structure parameters, such as the burst duration, the time between bursts, and the $\frac{1}{M}$ ambiguity factor corresponding to the whole- or fractional-cycle phase ambiguities, can also be set. The simulator generates random realizations of $\phi_{r}(t)$ based on these input parameters.

Second, the simulation and test environment applies the reconstruction technique and evaluates the performance of this technique on the simulated signal. The performance of the technique is evaluated by two metrics: (1) the percentage of ambiguities it is able to resolve correctly, and (2) the coherence time $\tau_{\text {coh }}$ of the difference between the smoothed reconstructed phase time history $\phi_{s}(t)$ and the ideal phase time history $\phi_{\text {ideal }}(t)$. Because it simulates the TDMA signals, the simulation and test environment has access to $\phi_{\text {ideal }}(t)$. The environment allows a user to vary the input parameters to explore the sensitivity of the reconstruction technique to each parameter in terms of these two metrics.

Sensitivity tests are used to determine the parameter space within which successful phase reconstruction is possible. Results for input parameters modeling a TDMA satellite communication system and the insight behind them are given in Sec. V.

\section{A. Error Source Modeling}

The magnitude of the error variations in the residual carrier phase $\phi_{r}(t)$ directly affects the performance of the reconstruction technique, which leads to errors in the smoothed reconstructed phase $\phi_{s}(t)$. The magnitude of error variations in $\phi_{s}(t)$ directly affects the usefulness of the TDMA signals for opportunistic navigation. Consequently, it is important to simulate TDMA signals such that they accurately reflect realworld systems. To correctly simulate the residual carrier phase, the simulator must have accurate models for the phase variations caused by each error component of $\phi_{r}(t)$ as outlined in (1). Recall that variations in $\phi_{r}(t)$ are caused by a combination of variations in the independent signal error components, in 
particular, those brought on the by the transmitter and receiver clocks, the propagation environment, and the receiver's frontend.

Modeling the various error sources can be greatly simplified by exploiting two key facts: (1) the sources are, for practical purposes, statistically independent, and (2) the error components $r_{e}(t), \delta t_{R X}, \delta t_{T X}$, and $\epsilon_{p}(t)$ in (1) can all be realistically modeled as second-order Gauss-Markov processes. More precisely, their individual contribution to the overall residual phase can be represented by a combination of random-walkphase and random-walk-frequency noise. But these are nothing more than the basic components of a simple clock error model. Therefore, one can develop a unified noise model that treats the phase errors due to clocks, $\frac{c}{\lambda} \delta t_{R X}(t)$ and $\frac{c}{\lambda} \delta t_{T X}(t)$, and the phase errors due to range and propagation effects, $\frac{1}{\lambda} r_{e}(t)$ and $\epsilon_{p}(t)$, as instances of the same two-parameter noise model as is commonly used to model clock errors [14].

Let $S_{\phi}(f)$ be the single-sided power spectral density (PSD) of some statistically stationary phase error process $\phi(t) . S_{\phi}(f)$ can be expressed as

$$
\mathcal{S}_{\phi}(f)=4 \int_{0}^{\infty} \mathcal{R}_{\phi}(\tau) \cos (2 \pi f \tau) d \tau
$$

where $\mathcal{R}_{\phi}(\tau)=\mathbb{E}[\phi(t) \phi(t+\tau)]$ is the autocorrelation function of $\phi(t)$. A common clock error model approximates $S_{\phi}(f)$ by a frequency-weighted summation of power-law parameters, called $h$-parameters $h_{\alpha}$ [18]:

$$
\mathcal{S}_{\phi}(f)=\frac{\nu_{0}^{2}}{f^{2}} \sum_{\alpha=-2}^{2} h_{\alpha} f^{\alpha} \quad 0<f<f_{h}
$$

where

$\nu_{0} \quad$ is the nominal center frequency of the phase data, in Hertz, and

$f_{h} \quad$ is the maximum frequency at which $\mathcal{S}_{\phi}(f)$ is evaluated, typically corresponding to the Nyquist rate of the sampled phase error process $\phi(t)$.

When only $h_{-2}$ (corresponding to frequency random walk) and $h_{0}$ (corresponding to phase random walk) are nonzero, the five-parameter model in (26) reduces to the two-parameter (second-order Gauss-Markov) clock error model often invoked in Kalman filtering [14].

The components of $\phi_{r}(t)$ and of $\phi_{s}(t)$ that can be accurately captured by a PSD model of the form in (26) are discussed in more detail subsequently. Those components that are instead better modeled via alternative methods are discussed in Sec. IV-C.

\section{B. Processes Modeled by their Power Spectra}

The following components of the residual phase $\phi_{r}(t)$ can be approximated by a power-law PSD model of the form in (26):

1) $\delta t_{T X}$ and $\delta t_{R X}$, the transmitter and receiver clock phase error terms. These terms are well characterized by their Allan variance [19] which can be readily transformed into a power-spectral density that can be modeled by the two-parameter clock error model discussed above.

2) $r_{e}(t)$, the range error term. This term includes line-ofsight errors in the modeled receiver motion and transmitter motion. For stationary transmitters and receivers, this term is constant. For moving transmitters such as satellites, this term captures errors in the satellite ephemeris. For moving receivers, this term additionally accounts for errors in the receiver's prediction of its motion, e.g., errors in inertial measurement unit (IMU) outputs. The model in (26) serves as a reasonable approximation for the PSD of these effects.

3) $\epsilon_{p}$, the signal propagation-induced phase errors. These errors are typically induced by the atmosphere, e.g., the ionosphere and troposphere, and by multipath. The model in (26) serves as a reasonable approximation for the PSD of these effects.

The above error processes, arising from unrelated sources, are statistically independent. As a result, their individual power-law PSD models of the form in (26) can be combined by summation to form an aggregate PSD:

$$
\mathcal{S}_{\phi_{\text {total }}}=\mathcal{S}_{\phi_{1}}+\mathcal{S}_{\phi_{2}}+\cdots+\mathcal{S}_{\phi_{N}} \text {. }
$$

\section{Processes Modeled by Alternative Methods}

For the following components of $\phi_{r}(t)$ or $\phi_{s}(t)$ a power-law PSD model is not appropriate:

1) $\gamma_{0}$ and $\psi_{0}$, the initial transmitter and receiver carrier phase offsets. These terms do not need to be modeled because they are constant and so do not cause variations in $\phi_{r}(t)$ or $\phi_{s}(t)$.

2) $v_{\phi}$, the measurement noise term. This term is better characterized by the more-commonly-known carrier-tonoise ratio $C / N_{0}$ than by a power spectra model. There is a direct relationship between a signal's $C / N_{0}$ and the phase measurement noise. However, due to nonlinearity in the phase discriminator, there is not a direct functional relationship between the $C / N_{0}$ and the $h$-parameters, in particular, the $h_{2}$ parameter (corresponding to white phase noise), except in the limit as $C / N_{0}$ is large (phase errors small). Thus, within the simulator, the characterization and simulation of measurement noise is kept in terms of $C / N_{0}$.

3) $v_{\phi_{s}}$, the smoothed measurement noise term. This term represents the measurement noise remaining after smoothing. This is neither Gaussian nor white noise. The effect of this term has only been analyzed through simulation and has not been characterized in terms of $h$ parameters. However, within an opportunistic framework and for a relatively high $C / N_{0}$, this term has little effect on the achievable coherent integration time.

4) $\frac{1}{M}\left[\eta(t)-\hat{\eta}_{s}(t)\right]$, the ambiguity resolution error term. This term is a measure of how well the fractionalcycle ambiguities were resolved from burst to burst. The errors in this term are dependent on how well the reconstruction technique performs under the various 
TABLE I

Sensitivity Testing Transmitter and Receiver Clock Stability

\begin{tabular}{|c|c|c|c|}
\hline Type & Quality & $h_{-2}$ & $h_{0}$ \\
\hline TX Clock & High-quality OCXO & $5.5 \mathrm{e}-26$ & $3 \mathrm{e}-22$ \\
\hline RX Clock 1 & High-quality TCXO & $2.9 \mathrm{e}-21$ & $3.4 \mathrm{e}-21$ \\
\hline RX Clock 2 & Medium-quality TCXO & $2.9 \mathrm{e}-21$ & $2.5 \mathrm{e}-20$ \\
\hline RX Clock 3 & Low-quality TCXO & $2.9 \mathrm{e}-21$ & $5 \mathrm{e}-19$ \\
\hline
\end{tabular}

errors contributed by the terms in $\phi_{r}(t)$. Consequently, even though phase variations due to this ambiguity error term can be expressed in terms of its power spectrum, a functional relationship would need to be built up between its $h$-parameters and the noise characterizations ( $h$-parameter PSD models, $C / N_{0}$, etc.) of the terms in $\phi_{r}(t)$. Given the authors' current knowledge, it does not appear possible to develop an analytical relationship between the statistics of $\frac{1}{M}\left[\eta(t)-\hat{\eta}_{s}(t)\right]$ and those of the error processes.

\section{Monte-Carlo Sensitivity Testing}

This section presents simulation input parameter bounds beyond which the reconstruction technique will fail. Successful reconstruction is measured by two metrics: (1) by the percentage of phase ambiguities the technique is able to resolve correctly, and (2) by the coherence time of the reconstruction error defined by the difference $\phi_{s}(t)-\phi_{\text {ideal }}(t)$. Sensitivity testing was done by varying the input parameters around a set of parameters that represent signals transmitted by a satellite-based TDMA communication system.

\section{A. Sensitivity to Receiver Clock Quality}

Figs. 6-8 show the results for a successful phase reconstruction attempt, a moderately successful attempt, and a failed attempt. A 10-second signal was simulated having a burst duration $T_{b}$ of 8 milliseconds, a burst period $T_{p}$ of 90 milliseconds, a reasonably high carrier-to-noise ratio at $63-\mathrm{dB}$, an oven-controlled crystal oscillator (OCXO)-quality transmitter clock, and three different temperature-compensated crystal oscillator (TCXO)-quality receiver clocks. These parameters are characteristic of those from a satellite-based TDMA communication system. The stability of the transmitter clock and the three receiver clocks is outlined in Table I and is described using the two-parameter clock model discussed in Sec. IV-A. The range- and propagation-induced phase errors modeled by $r_{e}(t)$ and $\epsilon_{p}(t)$ were assumed negligible in comparison to the receiver clock errors and so were modeled through small, but inconsequential increases to the receiver clock $h_{-2}$ and $h_{0}$ values listed in Table I.

It can be deduced from the figures that the quality of the receiver clock has an important impact on the performance of the reconstruction technique. The intuition here is that because the receiver clock errors $\delta t_{R X}(t)$ are part of the system process noise as described in Sec. III-A2, these errors, if too large, will reduce the smoother's ability to trust phase and phase-rate information extracted from past and future burst measurements when attempting to resolve phase ambiguities. As a result, as

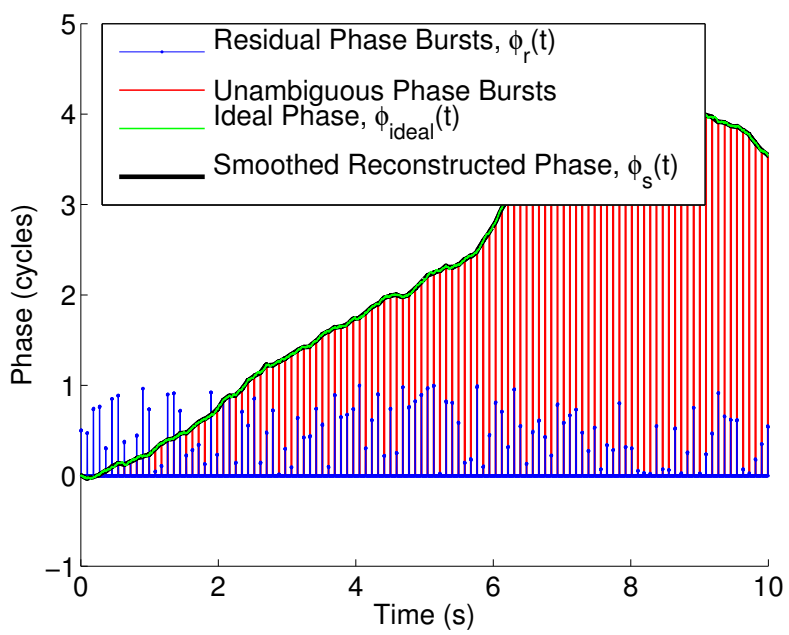

Fig. 6. A successful reconstruction of a simulated TDMA communication signal when using a high-quality TCXO as a receiver clock.

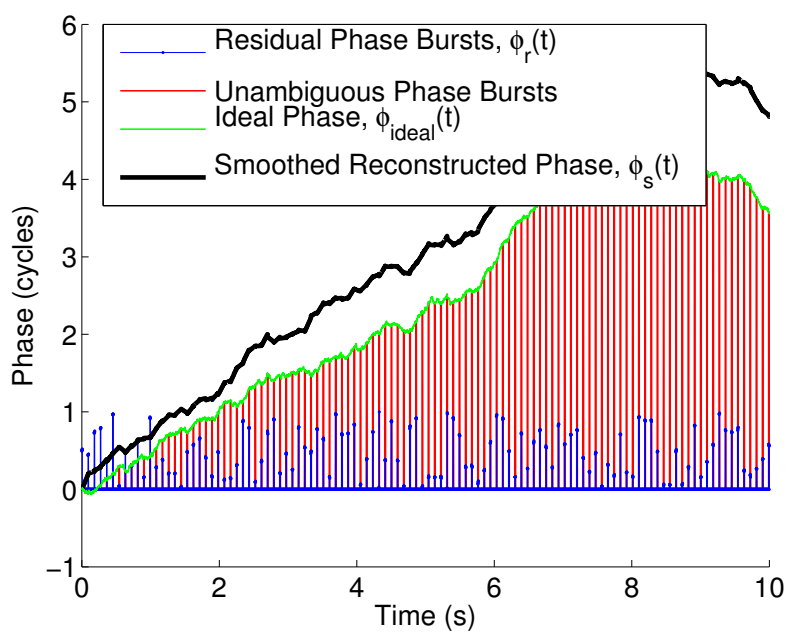

Fig. 7. A moderately successful reconstruction of a simulated TDMA communication signal when using a medium-quality TCXO as a receiver clock.

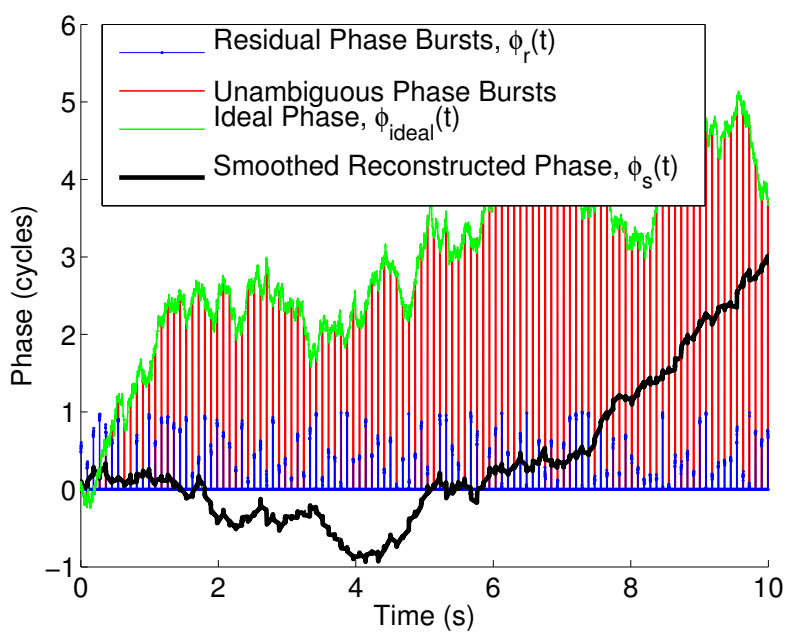

Fig. 8. A failed reconstruction of a simulated TDMA communication signal when using a low-quality TCXO as a receiver clock. 
TABLE II

RECONSTRUCTION PERFORMANCE RESULTS

\begin{tabular}{|c|c|c|}
\hline Quality of TCXO & Burst Error \% & Coherence Time (s) \\
\hline High & $0 \%$ & $>10$ \\
\hline Medium & $7 \%$ & $>10$ \\
\hline Low & $69 \%$ & 0.16 \\
\hline
\end{tabular}

process noise grows large, it becomes increasingly difficult to resolve these ambiguities.

Table II shows a summary of the performance results for the three receiver TCXOs. The table designates the performance of the technique in terms of the two metrics discussed above: the burst error percentage and the coherence time of the reconstruction error. It is important to note that although Fig. 7 shows that the phase was not reconstructed perfectly for the medium-quality TCXO, the error in the reconstructed phase time history still maintains a coherence time greater than 10 seconds, as shown in Table II. This is because the ambiguities were resolved correctly over $93 \%$ of the time. The 7\% of ambiguities resolved incorrectly were not enough to drastically disrupt the reconstruction technique and cause large reconstruction errors. Contrast this with the performance of the algorithm when a low-quality TCXO was used as the receiver clock (Fig. 8). In this scenario, the ambiguities were resolved incorrectly $69 \%$ of the time and the resulting phase error coherence time is 0.16 seconds, indicating a drastic breakdown in reconstruction.

\section{B. Sensitivity to the Combined $h_{0}$ Parameter and to the Burst Period $T_{p}$}

This section illustrates the sensitivity of the reconstruction technique to two parameters: (1) the combined random walk phase noise present within the residual carrier phase $\phi_{r}(t)$, and (2) the burst period $T_{p}$. Random walk phase noise is modeled by the $h_{0}$ power-law parameter presented in Sec. IV-A. It was found that the reconstruction algorithm is sensitive to the $h_{0}$ parameter and less sensitive to the other $h$-parameters. Thus only the $h_{0}$ parameter was varied during sensitivity testing while the others were held constant.

Figs. 9 and 10 illustrate the results of these tests. In Fig. 9, the coherence time of the reconstruction error is plotted as a function of increasing $h_{0}$. Different colored traces are used to represent different burst periods. The dotted traces represent the coherence time of the reconstructed phase time history with filtering but without smoothing whereas the solid traces depict the coherence time of the reconstructed phase with both filtering and smoothing. The waterfall structure of each trace indicates a breakdown point in the reconstruction technique. Around this point, the reconstruction algorithm begins to incorrectly resolve the phase ambiguities, leading to large errors in the reconstructed phase time history and, consequently, a drastic reduction in the coherence time.

Two features of Fig. 9 are worth noting. First, despite the justification for smoothing in Sec. III-F, the smoothing of the phase time history does not seem to drastically impact the overall error coherence time when compared against the

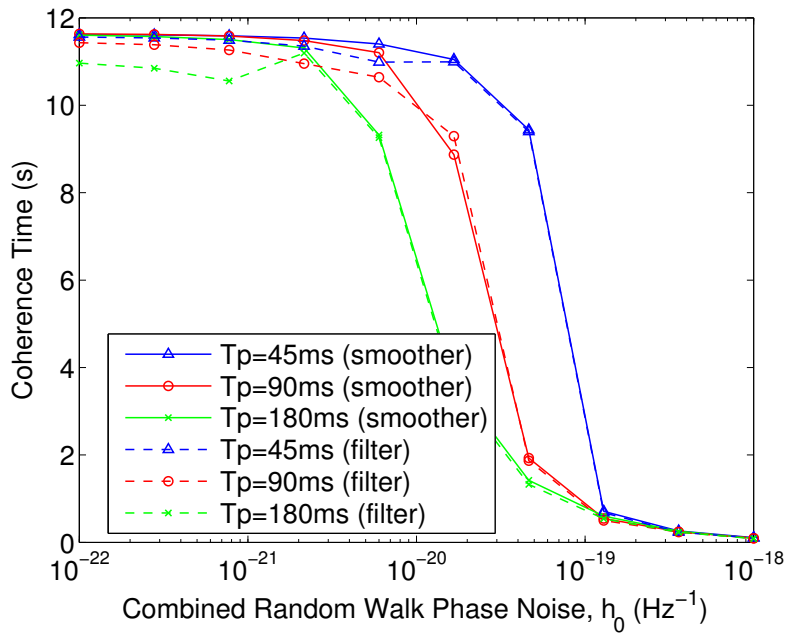

Fig. 9. Illustration of the coherence time of the error in the reconstructed signal as a function of the burst period $T_{p}$ and the $h_{0}$ power-law parameter representing the combined random walk phase noise present in the residual carrier phase $\phi_{r}(t)$.

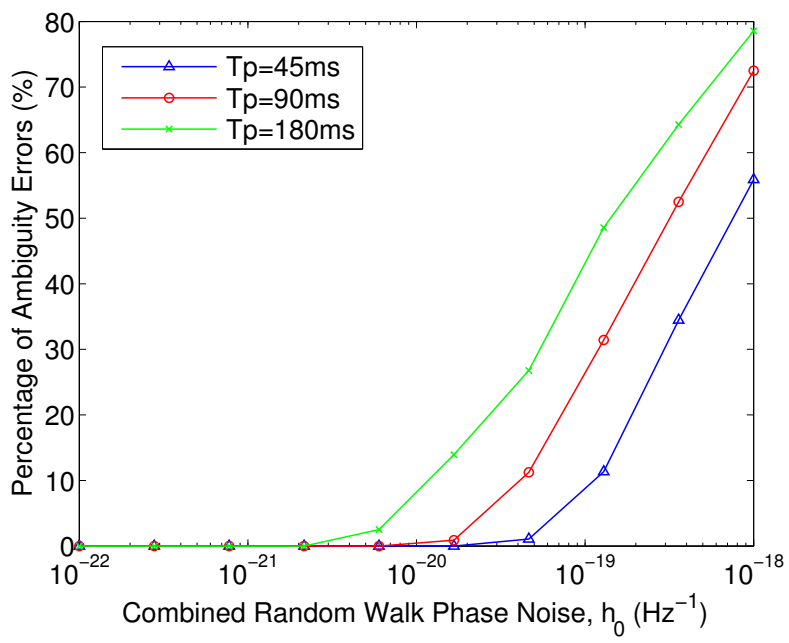

Fig. 10. Illustration of the percentage of ambiguity errors as a function of the burst period $T_{p}$ and the $h_{0}$ power-law parameter representing the combined random walk phase noise present in the residual carrier phase $\phi_{r}(t)$.

filtered phase time history. The filter-based traces largely overlap their filter-and-smoother-based counterparts. Therefore, for this particular setup, smoothing could be forgone in favor of a real-time reconstruction technique employing only the forward filter and the ambiguity resolution algorithm with little effect on the reconstruction performance. Second, attempts to reconstruct signals with a smaller burst period $T_{p}$ can sustain larger phase random walk errors, i.e., a larger combined $h_{0}$ value, before a breakdown occurs. This result is as would be expected because given a fixed $h_{0}$ value, a smaller $T_{p}$ makes it easier for the reconstruction algorithm to accurately estimate the phase trajectory between bursts and thus resolve the ambiguities at the beginning of each burst. This is best visualized in Fig. 4 where a smaller $T_{p}$ would leave less opportunity for the phase to drift unpredictably between bursts, 


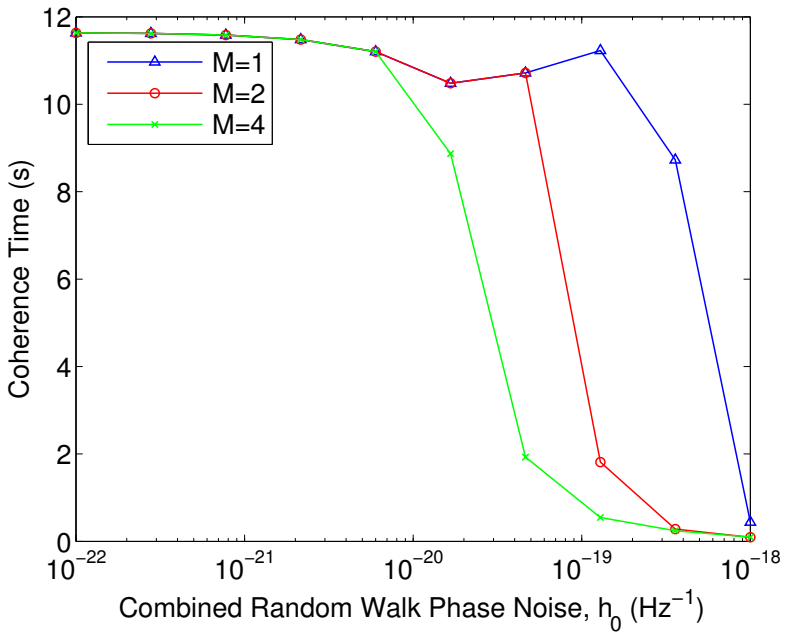

Fig. 11. Illustration of the coherence time of the error in the reconstructed signal as a function of the $\frac{1}{M}$ ambiguity factor and the $h_{0}$ power-law parameter representing the combined random walk phase noise present in the residual carrier phase $\phi_{r}(t)$.

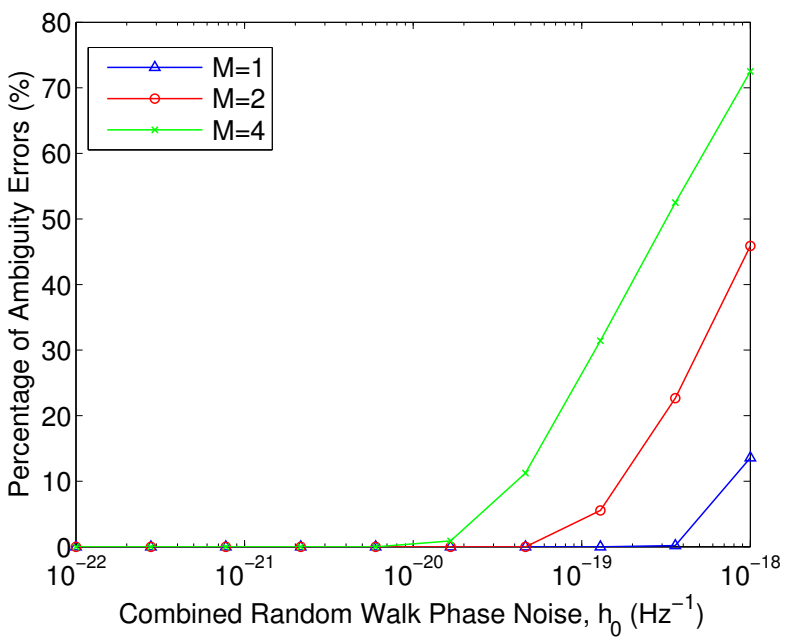

Fig. 12. Illustration of the percentage of ambiguity errors as a function of the $\frac{1}{M}$ ambiguity factor and the $h_{0}$ power-law parameter representing the combined random walk phase noise present in the residual carrier phase $\phi_{r}(t)$.

making it easier for the reconstruction technique to resolve the ambiguity.

Fig. 10 shows the percentage of ambiguity errors made over the entire reconstruction interval. Similar to the coherence time metric, each trace begins to rise quickly after a specific breakdown point in the reconstruction technique.

\section{Sensitivity to the Ambiguity Factor $\frac{1}{M}$}

Recall from Sec. II-A that the ambiguity factor $\frac{1}{M}$ is used to model the whole- or fractional-cycle phase ambiguities present at the beginning of each burst. Figs. 11 and 12 illustrate that a lower $M$ value leads to a higher $h_{0}$ breakdown point in both the coherence time and error probability performance metrics. Consequently, whole-cycle ambiguities $(M=1)$ are easier to

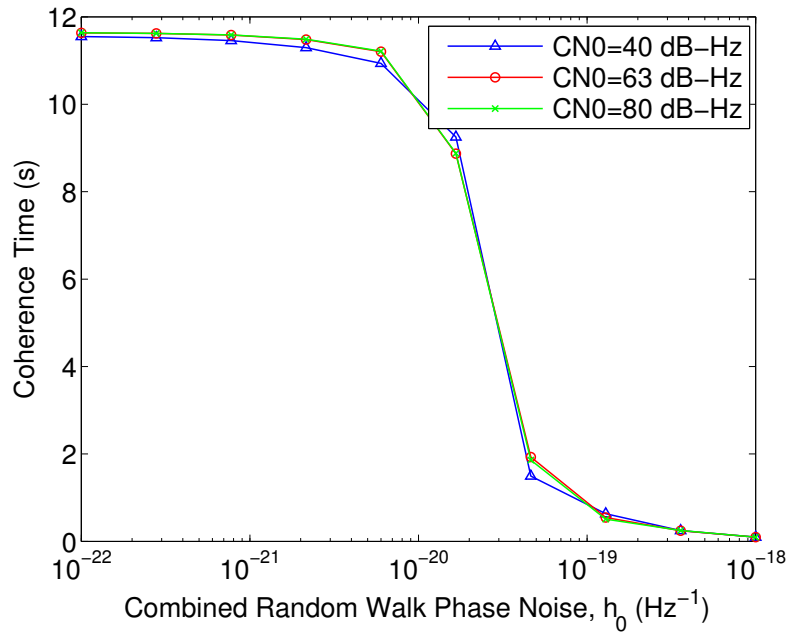

Fig. 13. Illustration of the coherence time of the error in the reconstructed signal as a function of $C / N_{0}$ and the $h_{0}$ power-law parameter representing the combined random walk phase noise present in the residual carrier phase $\phi_{r}(t)$.

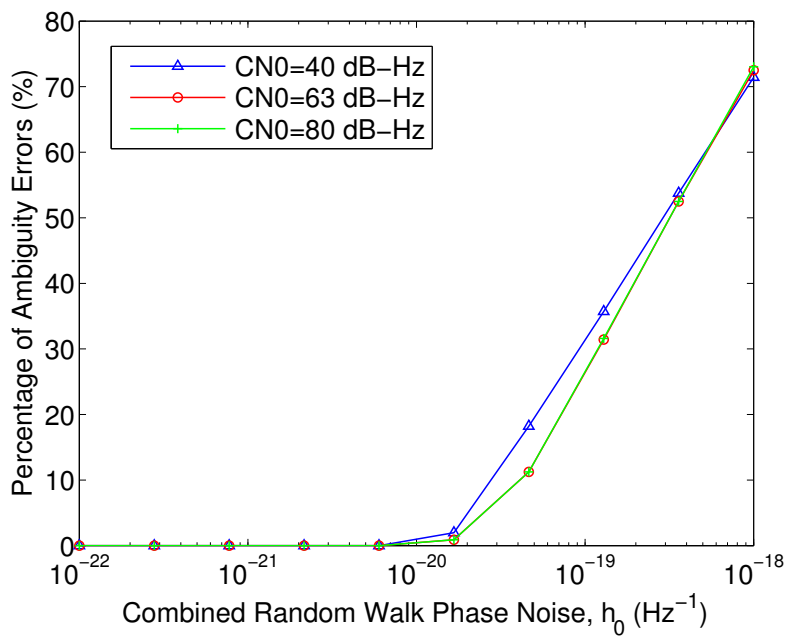

Fig. 14. Illustration of the percentage of ambiguity errors as a function of the $C / N_{0}$ and the $h_{0}$ power-law parameter representing the combined random walk phase noise present in the residual carrier phase $\phi_{r}(t)$.

resolve than half-cycle ambiguities $(M=2)$ which are easier to resolve than quarter-cycle ambiguities $(M=4)$, which was to be expected.

\section{Sensitivity to the Carrier-to-Noise Ratio $C / N_{0}$}

Figs. 13 and 14 illustrate the effect of $C / N_{0}$ on phase reconstruction. Somewhat surprisingly, $C / N_{0}$ was shown to have little effect on the breakdown point of the reconstruction technique. As described in Sec. IV-C, $C / N_{0}$ is related to white phase noise, i.e., the $h_{2}$ power-spectra parameter. It can be concluded that white phase noise in the received residual carrier phase $\phi_{r}(t)$ has little impact on the breakdown point of the reconstruction algorithm, within a range of reasonable carrier-to-noise ratios. 


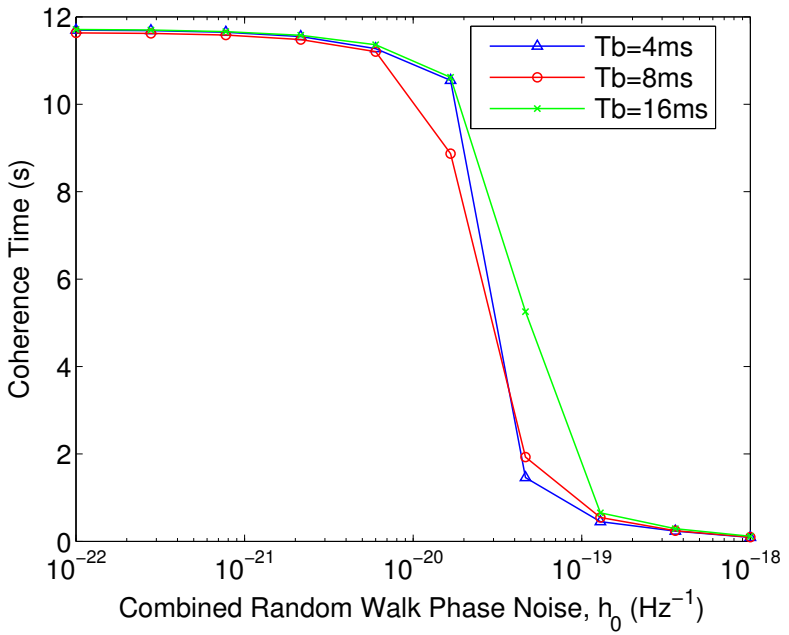

Fig. 15. Illustration of the coherence time of the error in the reconstructed signal as a function of the burst duration $T_{b}$ and the $h_{0}$ power-law parameter representing the combined random walk phase noise present in the residual carrier phase $\phi_{r}(t)$.

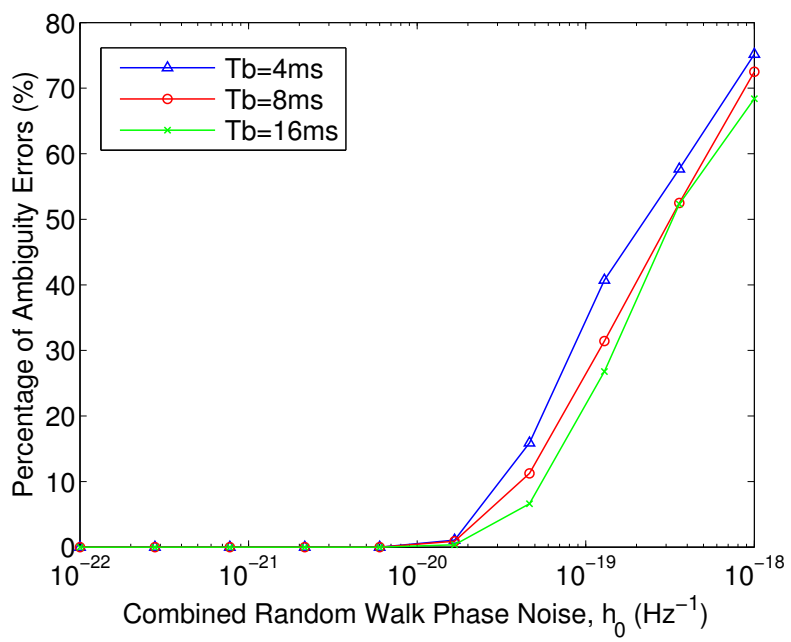

Fig. 16. Illustration of the percentage of ambiguity errors as a function of the burst duration $T_{b}$ and the $h_{0}$ power-law parameter representing the combined random walk phase noise present in the residual carrier phase $\phi_{r}(t)$.

\section{E. Sensitivity to the Burst Duration $T_{b}$}

Figs. 15 and 16 illustrate that although a longer burst duration does have some impact on the accuracy of the reconstruction technique, this impact is marginal. One explanation is that while a longer burst duration certainly improves the state estimates, particularly the estimate of phase-rate, during each burst, the ambiguity resolution algorithm does not derive much benefit from this phase-rate information. It is likely that for the range of parameters studied, the process noise arising from the error sources outlined in Sec. IV-B is substantial enough to cause large variations in the phase-rate from burst to burst. Consequently, when resolving the ambiguity for a given burst, exploiting the phase-rate information from the most recent past and future bursts is of little help.
TABLE III

STRUCTURAL PARAMETERS FOR THREE WIDESPREAD COMMUNICATION SYSTEMS [20], [21]

\begin{tabular}{|c|c|c|c|}
\hline System & Burst Duration, $T_{b}$ & Burst Period, $T_{p}$ & Ambiguity Factor, M \\
\hline SATCOM & $8 \mathrm{~ms}$ & $90-180 \mathrm{~ms}$ & $>1$ \\
\hline GSM & $101 \mu \mathrm{s}$ & $577 \mu \mathrm{s}$ & 1 \\
\hline LTE & $83 \mu \mathrm{s}$ & $5 \mathrm{~ms}$ & 1 \\
\hline
\end{tabular}

\section{F. Real-world Signal Parameters}

The sensitivity tests above were done using simulation input parameters characteristic of those from a satellitebased TDMA communication system. Receivers designed to incorporate signals from two other communication systems used today, (1) the Global System for Mobile (GSM) and (2) the 4G Long Term Evolution (LTE) system, could also employ this technique to reconstruct a continuous phase time history. GSM is a TDMA system. LTE, on the other hand, is an orthogonal frequency-division multiplexing (OFDM) system, where frequency division (rather than time division) is used to communicate with mobile receivers [20]. However, because LTE contains a known synchronization channel that is periodically transmitted over all frequencies for a small fraction of a subframe, this transmission of known bits can be thought of as a burst, during which the receiver can gain access to the underlying carrier phase. The reconstruction technique could be used to stitch together a complete phase time history between each synchronization channel. Table III outlines signal structural parameters for these two terrestrial communication systems as well as for the satellite-based communication system.

\section{CONCLUSION}

A technique to reconstruct a continuous phase time history from the noncontinuous phase bursts of time division multiple access (TDMA) signals has been developed. The technique combines an integer least squares method for estimating phase ambiguities at the beginning of each burst with a Kalman filter and smoother that correct for these ambiguities and optimally "stitch" the bursts together.

A Monte-Carlo-type simulation and test environment has been built in MATLAB to simulate TDMA signals, implement the reconstruction technique, and analyze the sensitivity of the technique to determine the parameter space within which successful reconstruction is possible. In this paper, sensitivity tests were performed through varying a set of simulation input parameters characterizing a satellite-based TDMA communication system. Simulation results indicate that successful carrier phase reconstruction is most strongly dependent on the burst period, the burst ambiguity factor, and on the combined phase random walk errors in the system. Results also indicate, somewhat counter-intuitively, that successful reconstruction is only weakly dependent on the signal burst duration and the carrier-to-noise ratio. 


\section{ACKNOWLEDGMENT}

This work was generously supported in part by the Department of Defense through the National Defense Science and Engineering Graduate (NDSEG) Fellowship Program.

\section{REFERENCES}

[1] J. Do, M. Rabinowitz, and P. Enge, "Performance of hybrid positioning system combining GPS and television signals," in Position, Location, And Navigation Symposium, IEEE/ION, 2006, pp. 556-564.

[2] K. Pesyna, Z. Kassas, J. Bhatti, and T. E. Humphreys, "Tightly-coupled opportunistic navigation for deep urban and indoor positioning," in Proceedings of the ION GNSS Meeting. Portland, Oregon: Institute of Navigation, 2011.

[3] M. Mathews, P. MacDoran, and K. Gold, "SCP enabled navigation using signals of opportunity in GPS obstructed environments," NAVIGATION, Journal of the Institute of Navigation, vol. 58, no. 2, pp. 91-110, 2011.

[4] P. Thevenon, S. Damien, O. Julien, C. Macabiau, M. Bousquet, L. Ries, and S. Corazza, "Positioning using mobile TV based on the dvb-sh standard," NAVIGATION, Journal of the Institute of Navigation, vol. 58, no. 2, pp. 71-90, 2011.

[5] M. Joerger, L. Gratton, B. Pervan, and C. Cohen, "Analysis of Iridiumaugmented GPS for floating carrier phase positioning," NAVIGATION, Journal of the Institute of Navigation, vol. 57, no. 2, pp. 137-160, 2010.

[6] L. Dai, Z. Wang, J. Wang, and Z. Yang, "Positioning with OFDM signals for the next-generation GNSS," Consumer Electronics, IEEE Transactions on, vol. 56, no. 2, pp. 374-379, 2010.

[7] C. Mensing and A. Dammann, "Positioning with OFDM based communications systems and GNSS in critical scenarios," in Proceedings of the 5th Workshop on Positioning, Navigation and Communication. IEEE, 2008, pp. $1-7$.

[8] D. Serant, O. Julien, L. Ries, P. Thevenon, and M. Dervin, "The Digital TV Case: Positioning using Signals-of-Opportunity based on OFDM Modulation," Inside GNSS, pp. 54-62, November/December 2011.

[9] P. J. Duffett-Smith and B. Tarlow, "E-GPS: Indoor mobile phone positioning on GSM and W-CDMA," in Proceedings of the ION GNSS Meeting. Long Beach, California: Institute of Navigation, 2005, pp. 2762-2768.

[10] M. Psiaki and S. Mohiuddin, "Modeling, analysis, and simulation of GPS carrier phase for spacecraft relative navigation," Journal of Guidance Control and Dynamics, vol. 30, no. 6, p. 1628, 2007.

[11] S. Khanafseh and B. Pervan, "New approach for calculating position domain integrity risk for cycle resolution in carrier phase navigation systems," IEEE Transactions on Aerospace and Electronic Systems, vol. 46, no. 1, pp. 296-307, 2010.

[12] G. J. Bierman, Factorization Methods for Discrete Sequential Estimation. New York: Academic Press, 1977.

[13] M. Psiaki, "Kalman filtering and smoothing to estimate real-valued states and integer constants," Journal of Guidance, Control, and Dynamics, vol. 33, no. 5, pp. 1404-1417, Sept.-Oct. 2010.

[14] R. G. Brown and P. Y. Hwang, Introduction to Random Signals and Applied Kalman Filtering. Wiley, 1997.

[15] M. Psiaki and S. Mohiuddin, "Global positioning system integer ambiguity resolution using factorized least-squares techniques," Journal of Guidance, Control, and Dynamics, vol. 30, no. 2, pp. 346-356, MarchApril 2007.

[16] P. De Jonge and C. Tiberius, "The LAMBDA method for integer ambiguity estimation: implementation aspects," Publications of the Delft Computing Centre, LGR-Series, 1996.

[17] P. Teunissen, P. De Jonge, and C. Tiberius, "Performance of the LAMBDA method for fast GPS ambiguity resolution," NAVIGATION, Journal of the Institute of Navigation, vol. 44, no. 3, pp. 373-383, 1997.

[18] A. Thompson, J. Moran, and G. Swenson, Target Interferometry and Synthesis in Radio Astronomy. Wiley, 2001, ch. 9: Very-Long-Baseline Interferometry, pp. 304-382.

[19] D. Allan, N. Ashby, C. Hodge, and H.-P. Company, The science of timekeeping. Hewlett-Packard, 1997.

[20] W. Xu and K. Manolakis, "Robust synchronization for 3GPP LTE system," in Global Telecommunications Conference (GLOBECOM 2010), 2010 IEEE, Dec. 2010, pp. $1-5$.

[21] C. Drane, M. Macnaughtan, and C. Scott, "Positioning GSM telephones," Communications Magazine, IEEE, vol. 36, no. 4, pp. 46-54, 1998. 